\title{
Packet Size Distribution: an Aside?
}

\author{
György Dán, Viktória Fodor and Gunnar Karlsson \\ KTH, Royal Institute of Technology, \\ Department of Microelectronics and Information Technology \\ \{gyuri, viktoria, gk\}@imit.kth.se
}

\begin{abstract}
For multimedia traffic like VBR video, knowledge of the average loss probability is not sufficient to determine the impact of loss on the perceived visual quality and on the possible ways of improving it, for example by forward error correction (FEC) and error concealment. In this paper we investigate how the packet size distribution affects the packet loss process, the distribution of the number of packets lost in a block of packets and the related FEC performance. We present an exact mathematical model for the loss process of an MMPP+ $M / E_{r} / 1 / K$ queue and compare the results of the model to simulations performed with various other packet size distributions (PSDs), among others, the measured PSD from an Internet backbone. We conclude that the packet size distribution affects the packet loss process and thus the efficiency of FEC. This conclusion is mainly valid in access networks where a single multimedia stream might affect the multiplexing behavior. The results show that analytical models of the PSD matching the first three moments (mean,variance and skewness) of the empirical PSD can be used to evaluate the performance of FEC in real networks. We also conclude that the exponential PSD, though it is not a worst case scenario, is a good approximation for the PSD of today's Internet to evaluate FEC performance.
\end{abstract}

\section{Introduction}

For flow-type multimedia communications, as opposed to elastic traffic, the average packet loss is not the only measure of interest. The burstiness of the loss process, the number of losses in a block of packets, has a great impact both on the user-perceived visual quality and on the possible ways of improving it, for example by error concealment and forward error correction.

Forward error correction (FEC) is an attractive means to decrease the loss probability experienced by delay sensitive traffic, such as real-time multimedia, when ARQ schemes can not be used to recover losses due to strict delay constraints. There are two main directions of FEC design to recover from packet losses. One solution, proposed by the IETF and implemented in Internet audio tools is to add a redundant copy of the original packet to one of the subsequent packets [1]. The other set of solutions, considered in this paper, use block coding schemes based on algebraic coding, e.g. Reed-Solomon coding [2]. The error correcting capability of RS codes with $k$ data packets and $c$ redundant packets is $c$ if data is lost. Thus, the capability of FEC to recover from losses depends on the distribution of the number of packets lost in a block, e.g. the burstiness of the loss process. 
The burstiness of the loss process in the network can be influenced by three factors, the burstiness of the stream traversing the network, the burstiness of the background traffic and the packet size distribution. The effects of the burstiness of the stream traversing the network and the background traffic have been investigated before [2-5]. The effects of the packet size distribution are not clear however. It is well known that in an $\mathrm{M} / \mathrm{G} / 1$ queue the average number of customers is direct proportional to the coefficient of variation $(\mathrm{CoV})$ of the service time distribution, as given by the Pollaczek-Khinchine formula [6]. For the finite capacity M/G/1 queue there is no closed form formula to calculate the packet loss probability $[7,8]$, though we know from experience that a lower $\mathrm{CoV}$ of the service time distribution yields lower average loss probability. It is however unclear how the distribution of the service time affects the loss process in a finite queue and thus how the potential of using FEC changes. The packet size distribution (PSD) in the network can vary on the short term due to changes in the ongoing traffic and on the long term as new applications and protocols emerge. As individual applications can not control the PSD in the network, it is important to know how the PSD will affect their performance, e.g. how much gain can an application expect from FEC given a certain measured end-to-end average loss probability.

In this paper we present a model to analyze the packet loss process of a bursty source, for example VBR video, multiplexed with background traffic in a single multiplexer with a finite queue and Erlang-r distributed packet sizes. We model the bursty source by an L-state Markov-modulated Poisson process (MMPP) while the background traffic is governed by a Poisson process. We compare the results of the model to the results of a model with deterministic packet sizes and to various simulations performed with general PSDs, among them the measured PSD of an Internet backbone [9], and investigate the effects of the network PSD on the packet loss process and the efficiency of FEC.

It is well known that compressed multimedia, like VBR video, exhibits a self-similar nature [10]. Yoshihara et al. use the superposition of 2-state IPPs to model self-similar traffic in [11] and compare the loss probability of the resulting $M M P P / D / 1 / K$ queue with simulations. They found that the approximation works well under heavy load conditions and gives an upper bound on the packet loss probabilities. Ryu and Elwalid [12] showed that short term correlations have dominant influence on the network performance under realistic scenarios of buffer sizes for real-time traffic. Thus the MMPP may be a practical model to derive approximate results for the queuing behavior of long range dependent traffic such as real-time VBR video, especially in the case of small buffer sizes [13]. Recently Cao et al. [14] showed that the traffic generated by a large number of sources tends to Poisson as the load increases due to statistical multiplexing and hence justifying the Poisson model for the background traffic. Recent measurements indicate that Internet traffic can be approximated by a non-stationary Poisson process [15]. According to the results the change free intervals are well above $150 \mathrm{~ms}$, the ITU's G.114 recommendation for end-to-end delay for real-time applications.

The paper is organized as follows. Section 2 gives an overview of the previous work on the modeling of the loss process of a single server queue. In Section 3 we describe our model used for calculating the loss probabilities in a block of packets. In Section 4 we evaluate the effects of the PSD on the packet loss process in various scenarios. We 
consider constant average load in Subsection 4.1, constant average loss probability in Subsection 4.2, and we isolate the effect of the PSD from other factors in Subsection 4.3. We conclude our work in Section 5.

\section{Related work}

In [16], Cidon et al. presented an exact analysis of the packet loss process in an $\mathrm{M} / \mathrm{M} / 1 / \mathrm{K}$ queue, that is the probability of losing $j$ packets in a block of $n$ packets, and showed that the distribution of losses may be bursty compared to the assumption of independence. They also considered a discrete time system fed with a Bernoulli arrival process describing the behavior of an ATM multiplexer. Gurewitz et al. presented explicit expressions for the above quantities of interest for the $\mathrm{M} / \mathrm{M} / 1 / \mathrm{K}$ queue in [17]. In [18], Altman et al. obtained the multidimensional generating function of the probability of $j$ losses in a block of $n$ packets and gave an easy-to-calculate asymptotic result under the condition that $n \leq K+j+1$.

Schulzrinne et al. [19] derived the conditional loss probability (CLP) for the $N *$ $I P P / D / 1 / K$ queue and showed that the CLP can be orders of magnitude higher than the loss probability. In [2] Kawahara et al. used an interrupted Bernoulli process to analyze the performance of FEC in a cell switched environment. The loss process of the $M M P P / D / 1 / K$ queue was analyzed in [20] and the results compared to a queue with exponential packet size distribution.

Models with general service time distribution have been proposed for calculating various measures of queuing performance [21,22], but not to analyze the loss process. Though models with exponential and deterministic PSDs are available, a thorough analysis of the effects of the PSD on the packet loss process has not yet been done.

\section{Model description}

Flows traversing large networks like the Internet cross several routers before reaching their destination. However, most of the losses in a flow occur in the router having the smallest available bandwidth along the transmission path, so that one may model the series of routers with a single router, the bottleneck [23, 24].

We model the network with a single queue with Erlang-r distributed packet sizes having average transmission time $1 / \mu$. The Erlang-r distribution is the distribution of the sum of $r$ independent identically distributed random variables each having an exponential distribution. By increasing $r$ to infinity the variance of the Erlang-r distribution goes to zero, and thus the distribution becomes deterministic.

Packets arrive to the system from two sources, a Markov-modulated Poisson process (MMPP) and a Poisson process, representing the tagged source and the background traffic respectively. The packets are stored in a buffer that can host up to K packets, and are served according to a FIFO policy. Every $n$ consecutive packets from the tagged source form a block, and we are interested in the probability distribution of the number of lost packets in a block in the steady state of the system. Throughout this section we use notations similar to those in [16]. 
We assume that the sources feeding the system are independent. The MMPP is described by the infinitesimal generator matrix $Q$ with elements $r_{l m}$ and the arrival rate matrix $\Lambda=\operatorname{diag}\left\{\lambda_{1}, \ldots, \lambda_{L}\right\}$, where $\lambda_{l}$ is the average arrival rate while the underlying Markov chain is in state $l$ [25]. The Poisson process modeling the background traffic has average arrival rate $\lambda$. The superposition of the two sources can be described by a single MMPP with arrival rate matrix $\hat{\Lambda}=\Lambda \oplus \lambda=\Lambda+\lambda I=\operatorname{diag}\left\{\hat{\lambda_{1}}, \ldots, \hat{\lambda_{L}}\right\}$, and infinitesimal generator $\hat{Q}=Q$, where $\oplus$ is the Kronecker sum. Packets arriving from both sources have the same size distribution. Each packet in the queue corresponds to $r$ exponential stages, and the state space of the queue is $\{0, \ldots, r K\} \times\{1, \ldots, L\}$.

Our purpose is to calculate the probability of $j$ losses in a block of $n$ packets $P(j, n)$, $n \geq 1,0 \leq j \leq n$. We define the probability $P_{i, l}^{a}(j, n), 0 \leq i \leq r K, l=1 \ldots L, n \geq 1,0 \leq$ $j \leq n$ as the probability of $j$ losses in a block of $n$ packets, given that the remaining number of exponential stages in the system is $i$ just before the arrival of the first packet in the block and the first packet of the block is generated in state $l$ of the MMPP. As the first packet in the block is arbitrary,

$$
P(j, n)=\sum_{l=1}^{L} \sum_{i=0}^{r K} \Pi(i, l) P_{i, l}^{a}(j, n) .
$$

$\Pi(i, l)$, the steady state distribution of the exponential stages in the queue as seen by an arriving packet can be derived from the steady state distribution of the $M M P P / E_{r} / 1 / K$ queue as

$$
\Pi(i, l)=\frac{\pi(i, l) \lambda_{l}}{\sum_{l=1}^{L} \lambda_{l} \sum_{i=0}^{r K} \pi(i, l)},
$$

where $\pi(i, l)$ is the steady state distribution of the $M M P P / E_{r} / 1 / K$ queue.

The probabilities $P_{i, l}^{a}(j, n)$ can be derived according to the following recursion. The recursion is initiated for $\mathrm{n}=1$ with the following relations

$$
\begin{aligned}
& P_{i, l}^{a}(j, 1)= \begin{cases}1 & j=0 \\
0 & j \geq 1\end{cases} \\
& P_{i, l}^{a}(j, 1)=\left\{\begin{array}{ll}
0 & j=0, j \geq 2 \\
1 & j=1
\end{array} \quad r(K-1)<i .\right.
\end{aligned}
$$

Using the notation $p_{m}=\frac{\lambda_{m}}{\lambda_{m}+\lambda}$ and $\bar{p}_{m}=\frac{\lambda}{\lambda_{m}+\lambda}$, for $n \geq 2$ the following equations hold.

$$
P_{i, l}^{a}(j, n)=\sum_{m=1}^{L} \sum_{k=0}^{i+r} Q_{i+r, l m}(k)\left\{p_{m} P_{i+r-k, m}^{a}(j, n-1)+\bar{p}_{m} P_{i+r-k, m}^{s}(j, n-1)\right\}
$$

for $0 \leq i \leq r(K-1)$, and for $r(K-1)<i$

$$
P_{i, l}^{a}(j, n)=\sum_{m=1}^{L} \sum_{k=0}^{i} Q_{i, l m}(k)\left\{p_{m} P_{i-k, m}^{a}(j-1, n-1)+\bar{p}_{m} P_{i-k, m}^{s}(j-1, n-1)\right\} .
$$

$P_{i, l}^{s}(j, n)$ is given by

$$
P_{i, l}^{s}(j, n)=\sum_{m=1}^{L} \sum_{k=0}^{i+r} Q_{i+r, l m}(k)\left\{p_{m} P_{i+r-k, m}^{a}(j, n)+\bar{p}_{m} P_{i+r-k, m}^{s}(j, n)\right\},
$$


for $0 \leq i \leq r(K-1)$, and for for $r(K-1)<i$

$$
P_{i, l}^{s}(j, n)=\sum_{m=1}^{L} \sum_{k=0}^{i} Q_{i, l m}(k)\left\{p_{m} P_{i-k, m}^{a}(j, n)+\bar{p}_{m} P_{i-k, m}^{s}(j, n)\right\} .
$$

The probability $P_{i, l}^{s}(j, n), 0 \leq i \leq r K, l=1 \ldots L, n \geq 1,0 \leq j \leq n$ is the probability of $j$ losses in a block of $n$ packets, given that the remaining number of exponential stages in the system is $i$ just before the arrival of a packet from the background traffic and the MMPP is in state $l . Q_{i, l m}(k)$ denotes the joint probability of that the next arrival will be in state $m$ of the MMPP and that $k$ exponential stages out of $i$ will be completed before the next arrival from the joint arrival process given that the last arrival was in state $l$ of the MMPP. A way to calculate $Q_{i, l m}(k)$ is shown in the Appendix.

The procedure of computing $P_{i, l}^{a}(j, n)$ is as follows. First we calculate $P_{i, l}^{a}(j, 1), i=$ $0 \ldots r K$ from the initial conditions (3). Then in iteration $k$ we first calculate $P_{i, l}^{s}(j, k), k=$ $1 \ldots n-1$ using equations (6) and (7) and the probabilities $P_{i, l}^{a}(j, k)$, which have been calculated during iteration $k-1$. Then we calculate $P_{i, l}^{a}(j, k+1)$ using equations (4) and (5).

\section{Performance analysis}

In this section we show results obtained with the $M M P P+M / E_{r} / 1 / K$ model described in Section 3, the $M M P P+M / D / 1 / K$ model described in [20] and simulations. The average packet length of both the tagged and the background traffic is set to 454 bytes, which is the mean packet size measured on an Internet backbone [9]. Note that increasing the average packet length is equivalent to decreasing the link speed, and thus the particular fixed value of the average packet length does not limit the generality of the results presented here. The PDF, $\operatorname{CoV}(\sigma / m)$ and skewness $\left(\sum(X-m)^{3} / \sigma^{3}\right)$ parameters of the twelve considered PSDs are shown in Table 1. The G1 distribution is the measured PSD on a 2.5 Gbps Internet backbone link as given by the Sprint IP Monitoring project [9]. The considered link speeds are $10 \mathrm{Mbps}, 22.5 \mathrm{Mbps}$ and $45 \mathrm{Mbps}$. The queuing delay is set to around $1.5 \mathrm{~ms}$ in all cases, resulting in queue lengths from 5 to 20 packets depending on the link speed. Both in the analytical models and in the simulations we consider a 3 state MMPP, with an average bitrate of $540 \mathrm{kbps}$, arrival intensities $\lambda_{1}=116 / s, \lambda_{2}=274 / s, \lambda_{3}=931 / s$ and transition rates $r_{12}=0.12594, r_{21}=$ $0.25, r_{23}=1.97, r_{32}=2$. These values were derived from an MPEG-4 encoded video trace by matching the average arrival intensities in the three states of the MMPP with the average frame size of the I,P and B frames. The simulations were performed in ns-2, the simulation time was between 40 thousand and 400 thousand seconds (5-50 million packets from the tagged source).

We use two measures to compare the packet loss process, the probability of loosing $j$ packets in a block of $n$ packets. The first one is a commonly used measure of closeness, the Kullback-Leibler distance [26] defined for two distributions as

$$
d\left(p_{1}, p_{2}\right)=\sum_{j=0}^{n} P_{1}(j, n) \log _{2} \frac{P_{1}(j, n)}{P_{2}(j, n)},
$$




\begin{tabular}{|l|r|r|l|r|}
\hline Distribution & CoV & Skewness & PDF & Notation \\
\hline General 1 & 1.2 & 1.07 & $b(x)$ taken from [9], see Figure 1 & G1 \\
\hline General 2 & 1.2 & 1.07 & $b(x)=0.74 N(127,20)+0.26 N(1366,20)$ & G2 \\
\hline Phase type & 1.2 & 1.07 & $b(x)=0.54 E(5,26)+0.46 E(5,956)$ & G3 \\
\hline Exponential & 1 & 2 & $E(1,454)$ & M \\
\hline General 4 & 1 & $\sqrt{2}$ & $b(x)=0.79 N(219,1)+0.21 N(1331,1)$ & G4 \\
\hline General 5 & $1 / \sqrt{2}$ & 2 & $b(x)=0.85 N(321,1)+0.15 N(1229,1)$ & G5 \\
\hline Erlang-2 & $1 / \sqrt{2}$ & $\sqrt{2}$ & $E(2,454)$ & E2 \\
\hline General 6 & $1 / \sqrt{2}$ & $\sqrt{0.4}$ & $b(x)=0.65 N(219,1)+0.35 N(892,1)$ & G6 \\
\hline General 7 & $\sqrt{0.1}$ & $\sqrt{2}$ & $b(x)=0.79 N(379,1)+0.21 N(731,1)$ & G7 \\
\hline Erlang-10 & $\sqrt{0.1}$ & $\sqrt{0.4}$ & $E(10,454)$ & E10 \\
\hline General 8 & $\sqrt{0.1}$ & 0 & $b(x)=0.5 N(310,1)+0.5 N(598,1)$ & G8 \\
\hline Deterministic & 0 & 0 & $b(x)=\delta_{454}(x)$ & D \\
\hline
\end{tabular}

Table 1. Considered packet size distributions: coefficient of variation, skewness, PDF and notation in the figures. $N(m, \sigma)$ denotes a normal distribution with mean $m$ and variance $\sigma^{2} . E(r, 1 / \mu)$ denotes an $r$-stage Erlang distribution with mean $1 / \mu$.

The Kullback-Leibler distance is the same as the relative entropy of $p_{1}$ with respect to $p_{2}$. It is not a true metric, as it is not symmetric and does not satisfy the triangle inequality, but it is always non-negative and equals zero only if $p_{1}=p_{2}$.

The second measure is based on the gain that can be achieved by using FEC. Given the probabilities $P(j, n)$ the uncorrected loss probability for an $\mathrm{RS}(\mathrm{k}, \mathrm{c}+\mathrm{k})$ scheme can be calculated as

$$
P_{\text {loss }}^{k, c+k}=\frac{1}{c+k} \sum_{j=c+1}^{c+k} j P(j, c+k) .
$$

Based on the uncorrected packet loss probability we define the FEC gain as the ratio of the average loss probability without the use of FEC and the uncorrected loss probability when using FEC: $f(k, c+k)=P_{\text {loss }} / P_{\text {loss }}^{k, c+k}$.

\subsection{Constant average load case}

In this subsection we investigate the effects of the PSD on the packet loss process and the efficiency of FEC as a function of the average load in the network. Figure 2 shows the uncorrected packet loss probability for $\operatorname{FEC}(1,1), \operatorname{FEC}(10,11)$ and $\operatorname{FEC}(20,22)$ on a 10 Mbps link for the G1,G2, G3, M and D distributions. Figures 3 and 4 show the same results on a $22.5 \mathrm{Mbps}$ and a $45 \mathrm{Mbps}$ link. The figures show that results obtained with the G1, G2 and G3 distributions are practically the same (the difference is less than $5 \%$ ). This indicates that by matching the first three moments of a distribution one can derive accurate results in terms of average loss probability and FEC gain even for very low loss probabilities. In the following we will only use the G1 distribution out of these three distributions. Figures 5 and 6 show the Kullback-Leibler distance obtained with different PSDs on a 10 Mbps link for $P(j, 11)$ and $P(j, 22)$ respectively. Figures 7 and 8 show the FEC gain for the same scenarios. Comparing the figures we conclude that 
$\operatorname{FEC}(10,11)$ and $\operatorname{FEC}(20,22)$ are qualitatively similar, and thus in the following we will only show figures for $\operatorname{FEC}(20,22)$ for brevity. Comparing results obtained with PSDs having the same CoV but different skewness we can see that even though the skewness has an effect on the packet loss process (especially at low loss probabilities), the $\mathrm{CoV}$ of the PSD has the biggest impact on the efficiency of FEC. We draw the same conclusion by examining Figures 9, 10, 11 and 12 which show the Kullback-Leibler distance and the FEC gain on a $22 \mathrm{Mbps}$ and a $45 \mathrm{Mbps}$ link as a function of the average load for $P(j, 22)$ and $\operatorname{FEC}(20,22)$ respectively. Thus, analytically tractable PSD models (for example phase-type, which includes both the Erlang and the hyper-exponential distributions as special cases, and has an extensive literature [27-30]) can be used to derive approximate results for FEC performance by matching the first two, and accurate results by matching the first three moments of the empirical PSD. Furthermore as the $\mathrm{CoV}$ of the PSD in the network is bounded from above, applications can be given a lower bound on the achievable gain of using FEC independent of the packet size distribution in the network. Though for some networks the exponential PSD might fit, it is clear from the results that it does not represent a worst case scenario if the average packet size is not equal to the center of the domain of the PSD and thus the CoV of the PSD can exceed one. Nevertheless, the exponential PSD is a good approximation for the considered empirical PSD G1, and for other empirical PSDs to be found at [9]. This finding justifies the assumption of exponential service time distribution in earlier works on the efficiency of FEC [1, 16-18,31].

The difference between the results obtained at a particular average load with distributions having different $\mathrm{CoV}$ values is significant, up to one order of magnitude in terms of FEC gain in the considered scenarios, a lower $\mathrm{CoV}$ value yielding a less bursty loss process. The difference however is partly due to the different average loss probabilities. We eliminate the effects of the average loss probability in the following subsection.

\subsection{Constant average packet loss case}

In this subsection we consider results with different PSDs as a function of the average loss probability. This enables us to investigate what an application (unaware of the network PSD) can expect from FEC given that it experiences a certain end-to-end average packet loss probability. In order to be able to compare the packet loss process at a certain average loss probability we take the results from simulations with the G1 PSD and increase the background traffic of the mathematical models to match the average packet loss probability given by the simulations.

Figure 13 shows the Kullback-Leibler distance between the results obtained with the different distributions as a function of the average loss probability on a $10 \mathrm{Mbps}$ link for $P(j, 22)$. The figure shows that the distance between the results obtained with different distributions decreased significantly (three orders of magnitude). Figure 14 shows the FEC gain for the same scenario. The effects of the PSD are significantly smaller compared to Figure 8.

Figures 15 and 16 show the FEC gain on a 22.5 Mbps and a 45 Mbps link respectively. Comparing the figures we can see that the difference between results with different PSDs in terms of FEC gain decreases as the link speed increases (from $10 \mathrm{Mbps}$ to $45 \mathrm{Mbps}$ ). The reason for this is that the higher the link speed the less the background 
traffic has to be changed to keep the average loss probability constant, and thus the change in the level of statistical multiplexing decreases.

The observed difference in FEC gain can be due to the difference in the level of statistical multiplexing (the background traffic intensity was increased to maintain the average loss probability constant and as a result the packet loss process became more independent) and to the difference between the packet size distributions.

\subsection{Isolating the effects of the packet size distribution}

In this subsection we separate the effects of the level of statistical multiplexing and the PSD. We do it by changing the arrival intensity of both the background traffic and the tagged stream in the mathematical models in order to match the average loss probability given by the simulations with the G1 PSD, thus we keep the level of statistical multiplexing constant (doing so is equivalent to matching the average loss probability through decreasing the link speed). Figure 17 shows the Kullback-Leibler distance as a function of the average loss probability on a $10 \mathrm{Mbps}$ link for $P(j, 22)$. Comparing this to Figure 13 we can see a further significant decrease in the distance of the distributions. The same effect can be seen in Figure 18, which shows the FEC gain on a $10 \mathrm{Mbps}$ link as a function of the average loss probability for $\operatorname{FEC}(20,22)$. Thus the difference in the FEC gain considering a fixed average loss (Section 4.2) is mainly due to the different levels of statistical multiplexing and in a lower extent to the different PSD. This is in accordance with the observation in Section 4.2 that the difference between the results with different PSDs decreases as the link speed increases.

\section{Conclusion}

In this paper we investigated the effects of the packet size distribution on the packet loss process and the related FEC performance in a single server queue with a finite buffer. We presented a mathematical model for the analysis of the packet loss process of the $M M P P+M / E_{r} / 1 / K$ queue and compared the results of simulations and mathematical models in different scenarios. Our results show that analytical models of the PSD matching the first three moments of the empirical PSD can be used to evaluate the performance of FEC in real networks, while the exponential PSD is a reasonable approximation for the PSD of today's Internet to evaluate FEC performance. Nevertheless the exponential PSD is not a worst case scenario, the PSD in today's networks has a higher $\mathrm{CoV}$ and thus shows slightly worse queueing performance. However, as the $\mathrm{CoV}$ of the packet size distribution in a real network is bounded from above, one can give a lower bound on the efficiency of FEC and thus predict its performance. The results show that the effects of the packet size distribution decrease as the link speed increases if one considers a particular average packet loss probability. Thus at a given average loss probability the actual network PSD does not influence the efficiency of FEC on a backbone link, however it has a big influence on it in access networks. At the same time applications can have a bigger influence on the packet size distribution in access networks and thus have an impact on the packet loss process of their traffic. The results presented here can serve as a basis for future research on the performance of end-to-end error control and facilitate the use of FEC in tomorrow's applications. 

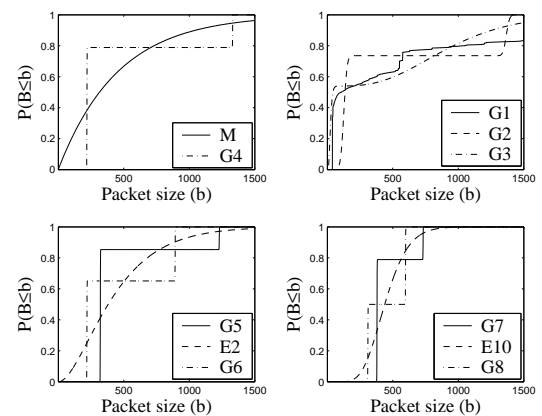

Fig. 1. Cumulative density functions of the considered packet size distributions.

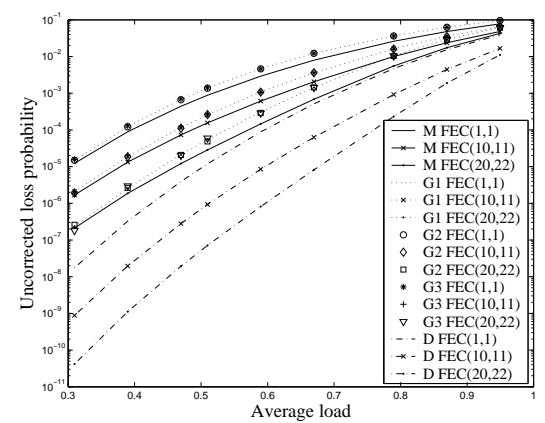

Fig. 3. Average loss probability with and without FEC vs average load on a 22.5 Mbps link.

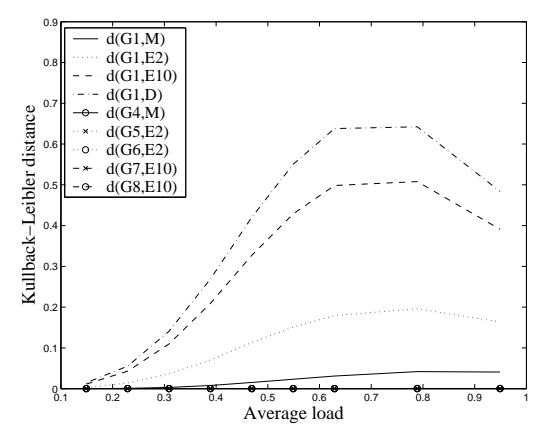

Fig. 5. Kullback-Leibler distance vs average load for $\mathrm{P}(\mathrm{j}, 11)$ on a $10 \mathrm{Mbps}$ link

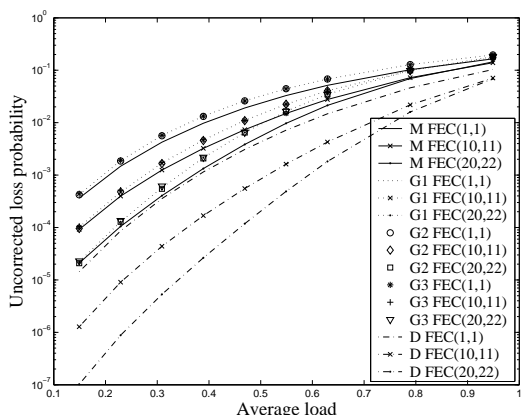

Fig. 2. Average loss probability with and without FEC vs average load on a $10 \mathrm{Mbps}$ link.

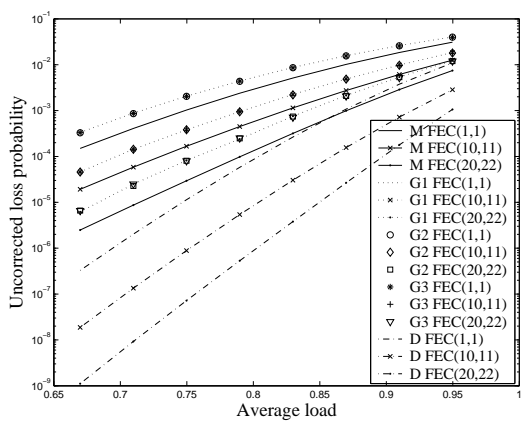

Fig. 4. Average loss probability with and without FEC vs average load on a $45 \mathrm{Mbps}$ link.

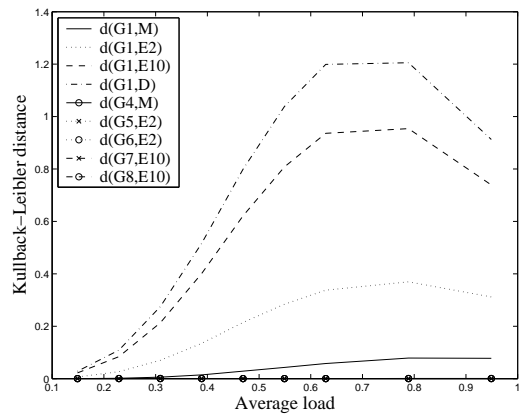

Fig. 6. Kullback-Leibler distance vs average load for $\mathrm{P}(\mathrm{j}, 22)$ on a $10 \mathrm{Mbps}$ link 


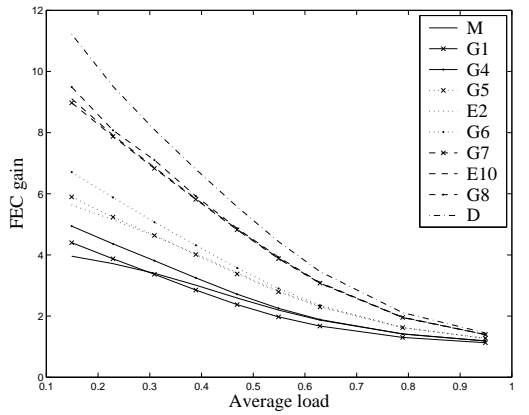

Fig. 7. FEC gain vs average load for FEC $(10,11)$ on a 10 Mbps link

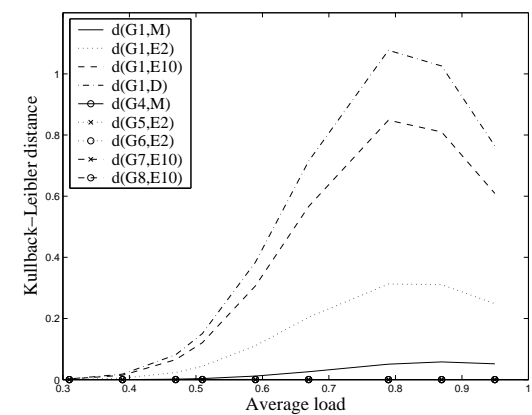

Fig. 9. Kullback-Leibler distance vs average load for $\mathrm{P}(\mathrm{j}, 22)$ on a $22.5 \mathrm{Mbps}$ link

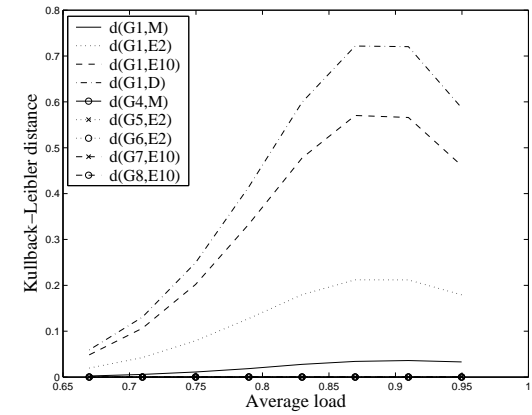

Fig. 11. Kullback-Leibler distance vs average load for $\mathrm{P}(\mathrm{j}, 22)$ on a $45 \mathrm{Mbps}$ link

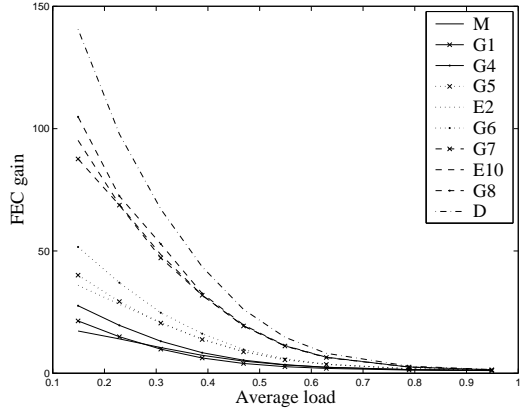

Fig. 8. FEC gain vs average load for $\operatorname{FEC}(20,22)$ on a $10 \mathrm{Mbps}$ link

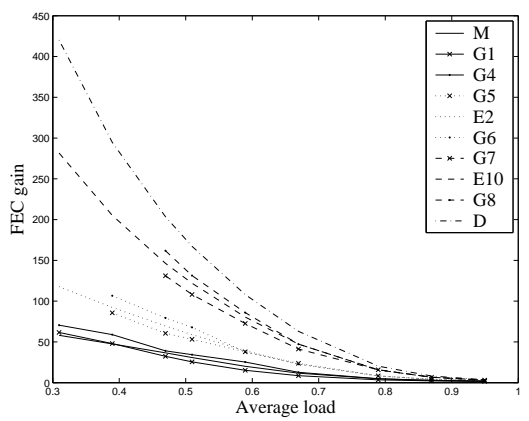

Fig. 10. FEC gain vs average load for FEC $(20,22)$ on a $22.5 \mathrm{Mbps}$ link

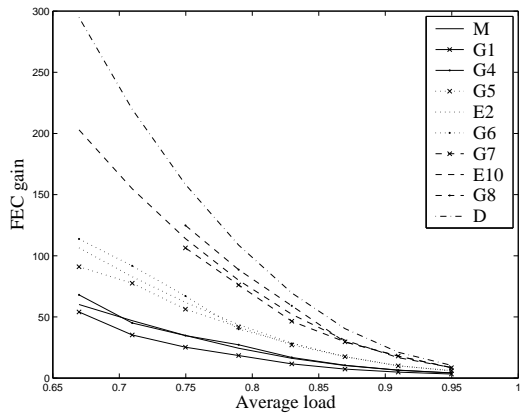

Fig. 12. FEC gain vs average load for $\operatorname{FEC}(20,22)$ on a 45 Mbps link 


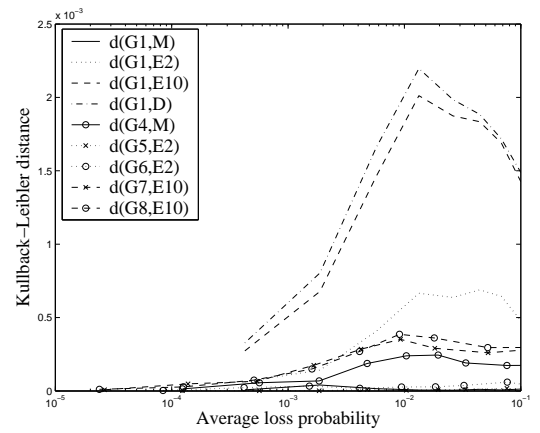

Fig. 13. Kullback-Leibler distance vs average loss probability for $\mathrm{P}(\mathrm{j}, 22)$ on a $10 \mathrm{Mbps}$ link

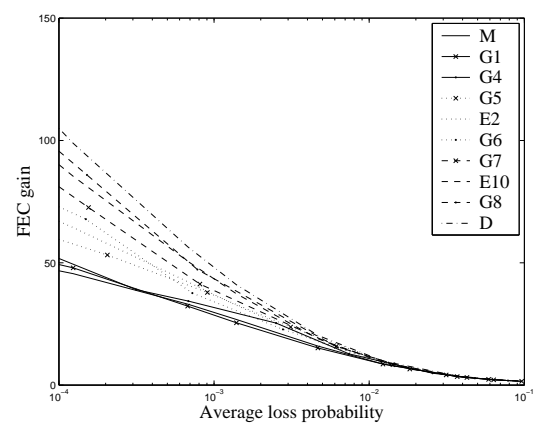

Fig. 15. FEC gain vs average loss probability for $\operatorname{FEC}(20,22)$ on a 22.5 Mbps link

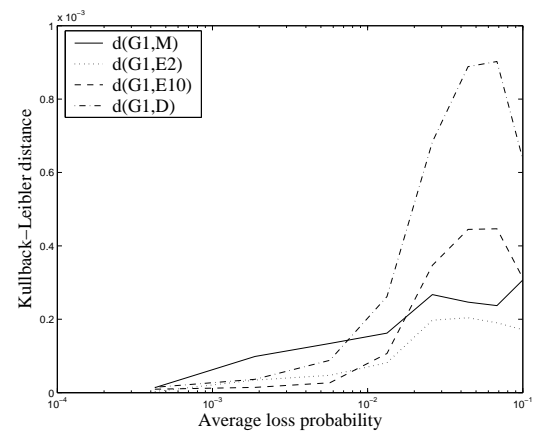

Fig. 17. Kullback-Leibler distance vs average loss probability for $\mathrm{P}(\mathrm{j}, 22)$ on a $10 \mathrm{Mbps}$ link (same level of statistical multiplexing)

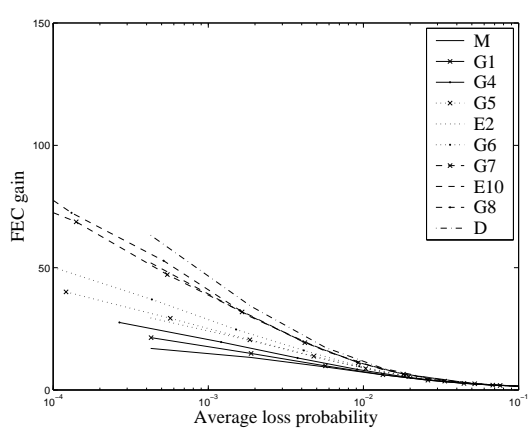

Fig. 14. FEC gain vs average loss probability for $\operatorname{FEC}(20,22)$ on a $10 \mathrm{Mbps}$ link

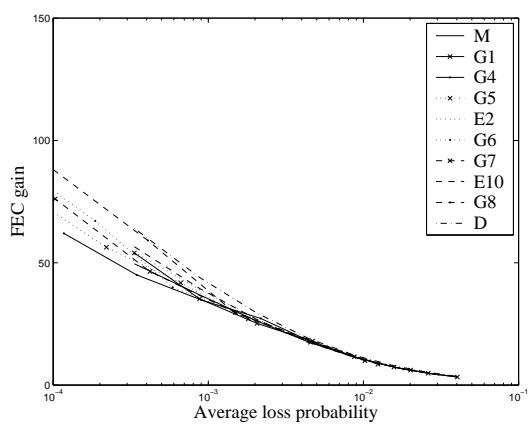

Fig. 16. FEC gain vs average loss probability for $\operatorname{FEC}(20,22)$ on a 45 Mbps link

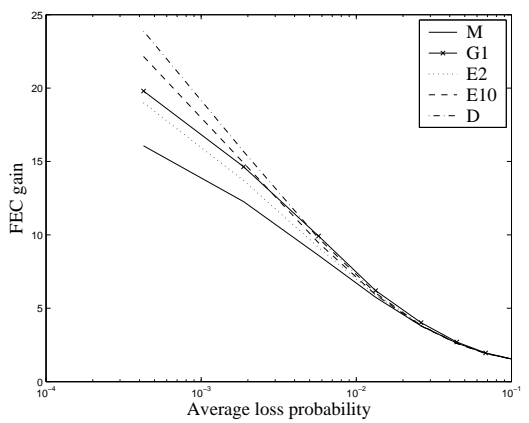

Fig. 18. FEC gain vs average loss probability for $\operatorname{FEC}(20,22)$ on a $10 \mathrm{Mbps}$ link (same level of statistical multiplexing) 


\section{Appendix}

The probability $Q_{i, l m}(k)$ denotes the joint conditional probability that between two arrivals from the joint arrival stream there are $k$ exponential stage completions out of $i$ and the state of the MMPP at the moment of the arrival is $m$ given that at the time of the last arrival the MMPP was in state $l . Q_{i, l m}(k)$ can be expressed as

$$
\begin{aligned}
& Q_{i}^{l, m}(k)=P^{l, m}(k) \quad \text { ifk }<i \\
& Q_{i}^{l, m}(k)=\sum_{j=i}^{\infty} P^{l, m}(j) \quad \text { if } k=i,
\end{aligned}
$$

where $P^{l, m}(k)$ denotes the joint probability of having $k$ exponential stage completions between two arrivals and the next arrival coming in state $m$ of the MMPP given that the last arrival came in state $l$.

The z-transform $P^{l, m}(z)$ of $P^{l, m}(k)$ is given by

$$
P^{l, m}(z)=\sum_{k=0}^{\infty}\left(\int_{0}^{\infty} \frac{(r \mu t)^{k}}{k !} e^{-r \mu t} f^{l, m}(t) d t\right) z^{k}=f^{l, m *}(r \mu-r \mu z)
$$

where $f^{l, m}(t)$ is the joint distribution of the interarrival-time and the probability that the next arrival is in state $m$ given that the last arrival was in state $l$ of the MMPP. The Laplace transform of $f^{l, m}(t)$ is denoted with $f^{l, m *}(s)$ and is given by

$$
f^{l, m *}(s)=\mathcal{L}\left\{e^{(\hat{Q}-\hat{\Lambda}) x} \hat{\Lambda}\right\}=(s I-\hat{Q}+\hat{\Lambda})^{-1} \hat{\Lambda}
$$

The inverse Laplace-transform of (12) and thus the inverse z-transform of (11) can be expressed analytically by partial fraction decomposition as long as $L \leq 4$, and has the form

$$
f^{l, m}(t)=\sum_{j=1}^{L} B_{j}^{l, m} e^{\beta_{j} t}
$$

where $\beta_{j}$ are the roots of $t(s)=\operatorname{det}[s I-\hat{Q}+\hat{\Lambda}]$. Using the substitution $\alpha_{j}=1+\beta_{j} / \mu$ and $A_{j}^{l, m}=B_{j}^{l, m} /\left(\mu \alpha_{j}\right)$ one can calculate $P^{l, m}(k)$ based on (11)

$$
P^{l, m}(k)=\sum_{j=1}^{L} A_{j}^{l, m} \frac{1}{\alpha_{j}^{k}}
$$

Given the probability $P^{l, m}(k)$ one can express $Q_{i}(k)$ as

$$
Q_{i}(k)=\left\{\begin{array}{l}
\sum_{j=1}^{L} A_{j}^{l m}\left(\frac{1}{\alpha_{j}}\right)^{k} \quad 0 \leq k<i \\
\sum_{j=1}^{L} \frac{A_{j}^{l m}}{1-1 / \alpha_{j}}\left(\frac{1}{\alpha_{j}}\right)^{i} k=i .
\end{array}\right.
$$

A more detailed description of the calculation of $Q_{i, l m}(k)$ can be found in [32]. 


\section{References}

1. P. Dube and E. Altman, "Utility analysis of simple FEC schemes for VoIP," in Proc. of Networking 2002, pp. 226-239, May 2002.

2. K. Kawahara, K. Kumazoe, T. Takine, and Y. Oie, "Forward error correction in ATM networks: An analysis of cell loss distribution in a block," in Proc. of IEEE INFOCOM, pp. 1150-1159, June 1994.

3. E. Biersack, "Performance evaluation of forward error correction in ATM networks," in Proc. of ACM SIGCOMM, pp. 248-257, August 1992.

4. G. Dán and V. Fodor, "Quality differentiation with source shaping and forward error correction," in Proc. of MIPS'03, pp. 222-233, November 2003.

5. P. Frossard, "FEC performances in multimedia streaming," IEEE Comm. Letters, vol. 5, no. 3, pp. 122-124, 2001.

6. L. Kleinrock, Queueing Systems, vol. I. Wiley, New York, 1975.

7. E. Altman, C. Barakat, and V. M. Ramos, "On the utility of FEC mechanisms for audio applications," in Proc. of Quality of Future Internet Services, LNCS 2156, pp. 45-56, 2001.

8. J. W. Cohen, The Single Server Queue. North-Holland Publishing, Amsterdam, 1969.

9. Sprint IP Monitoring Project, "http://ipmon.sprint.com/."

10. J. Beran, R. Sherman, M. Taqqu, and W. Willinger, "Long-range dependence in variablebit-rate video traffic," IEEE Trans. on Communications, vol. 43, no. 2/3/4, pp. 1566-1579, 1995.

11. T. Yoshihara, S. Kasahara, and Y. Takahashi, "Practical time-scale fitting of self-similar traffic with markov-modulated poisson process," Telecommunication Systems, vol. 17, no. 1-2, pp. 185-211, 2001

12. B. Ryu and A. Elwalid, "The importance of long-range dependence of VBR video traffic in ATM traffic engineering: Myths and realities," in Proc. of ACM SIGCOMM, pp. 3-14, 1996.

13. P. Skelly, M. Schwartz, and S. Dixit, "A histogram-based model for video traffic behavior in an ATM multiplexer," Trans. on Networking, vol. 1, pp. 446-458, August 1993.

14. J. Cao, W. S. Cleveland, D. Lin, and D. X. Sun, "Internet traffic tends toward poisson and independent as the load increases," in Nonlinear Estimation and Classification, Springer, 2002.

15. T. Karagiannis, M. Molle, and M. Faloutsos, "A nonstationary poisson view of internet traffic," in Proc. of IEEE INFOCOM, pp. 1-1, March 2004.

16. I. Cidon, A. Khamisy, and M. Sidi, "Analysis of packet loss processes in high speed networks," IEEE Trans. on Inform. Theory, vol. IT-39, pp. 98-108, Jan. 1993.

17. O. Gurewitz, M. Sidi, and I. Cidon, "The ballot theorem strikes again: Packet loss process distribution," IEEE Trans. on Inform. Theory, vol. IT-46, pp. 2599-2595, November 2000.

18. E. Altman and A. Jean-Marie, "Loss probabilities for messages with redundant packets feeding a finite buffer," IEEE Journal on Selected Areas in Comm., vol. 16, no. 5, pp. 779-787, 1998.

19. H. Schulzrinne, J. Kurose, and D. Towsley, "Loss correlation for queues with bursty input streams," in Proc. of IEEE ICC, pp. 219-224, 1992.

20. G. Dán, V. Fodor, and G. Karlsson, "Analysis of the packet loss process for multimedia traffic," in Proc. of the $12^{\text {th }}$ International Conference on Telecommunication Systems, Modeling and Analysis, July 2004.

21. H. Heffes and D. M. Lucantoni, "A markov modulated characterization of packetized voice and data traffic and related statistical multiplexer performance," IEEE Journal on Selected Areas in Comm., vol. 4, pp. 856-868, September 1986.

22. C. Blondia, "The N/G/1 finite capacity queue," Commun. Statist. - Stochastic Models, vol. 5, no. 2, pp. 273-294, 1989. 
23. J. C. Bolot, "End-to-end delay and loss behavior in the Internet," September 1993.

24. O. J. Boxma, "Sojourn times in cyclic queues - the influence of the slowest server," Computer Performance and Reliability, pp. 13-24, 1988.

25. W. Fischer and K. Meier-Hellstern, "The markov-modulated poisson process MMPP cookbook," Performance Evaluation, vol. 18, no. 2, pp. 149-171, 1992.

26. S. Kullback, Information Theory and Statistics. Wiley, New York, 1959.

27. L. Le Ny and B. Sericola, "Transient analysis of the BMAP/PH/1 queue," I.J. of Simulation, vol. 3, no. 3-4, pp. 4-15, 2003.

28. W. Whitt, "Approximating a point process by a renewal process, i: Two basic methods," Operations Research, vol. 30, no. 1, pp. 125-147, 1982.

29. M. Neuts, Matrix Geometric Solutions in Stochastic Models. John Hopkins University Press, 1981.

30. E. P. C. Kao, "Using state reduction for computing steady state probabilities of queues of GI/PH/1 types," ORSA J. Comput., vol. 3, no. 3, pp. 231-240, 1991.

31. O. Ait-Hellal, E. Altman, A. Jean-Marie, and I. A. Kurkova, "On loss probabilities in presence of redundant packets and several traffic sources," Performance Evaluation, vol. 36-37, pp. 485-518, 1999.

32. G. Dán, "Analysis of the loss process in an $\mathrm{MMPP}+\mathrm{M} / \mathrm{M} / 1 / \mathrm{K}$ queue," TRITA-IMIT-LCN R 04:02, KTH/IMIT/LCN, January 2004. 